\title{
DESIGN OF MILITARY ACTIONS IN THE OPERATIONAL ENVIRONMENT HYBRID TYPE
}

\author{
Mihai Marcel NEAG \\ "Nicolae Bălcescu" Land Forces Academy, Sibiu, Romania \\ mmneag@yahoo.com
}

\begin{abstract}
Collective defense arrangements promoted by security-related institutions have generally proven that they can produce the deterrent effect of the armed conflict. However, hybrid warfare actions have challenged the capability of countering and threatening hybrid threats. The paper seeks a point of view on developments in the current operational environment, focusing on the review of the military operations typology in identifying potential measures to counteract the actions of the hybrid war and highlighting the need for a new approach to the construction of armed power in terms of new types risks, challenges and threats.
\end{abstract}

Keywords: hybrid operating environment, multidimensional operational environment, comprehensive approach, military scenario

\section{Introduction}

Military events of this century have confirmed that armed struggle continues to be the means to resolve disputes between conflicting human communities. It is known that the point of maximum intensity of the confrontation is the military conflict, preceded by an upward path, marked by distinct stages of tension between the opponents and followed by a downward slope, returning to a redefined "normality" and gradually regained.

Recent conflicts demonstrate that in the current security environment, military operations have a common and multinational character, and conventional actions intertwine with unconventional and asymmetric ones. Whatever opponents we may report, such as state actors, non-state actors, or a combination of them, in a possible hybrid conflict will use a wide range of asymmetric actions to exploit the vulnerabilities of the opponent. This includes terrorist, insurgent, separatist, and organized crime as part of a dynamic and diversified mix of military-based analysts in the concept of "hybrid conflict".

If, in terms of military conflicts, we are talking about disimetry or non-symmetry as a term to structural and actional symmetry, non-military conflicts predominate asymmetries. Asymmetry is characterized by innovative behavior and adaptability to concrete situations created by conventional, technological and decision-making superiority.

Following the evaluation of the current operational environment we have found that the influence of the economic, political, diplomatic, informational and psychological aspects now occupies, more than ever, a very important place in the military operations architecture, but also the fact that the evolution of the technology in the field of communications, and cyber defense has led to an exponential increase in the power of information. We have noticed the ability of the economic 
environment to change any other trend resulting from the analysis of the operational environment, to guide and, if necessary, to dictate the direction and orientation of the diplomatic options of the states.

\section{Multidimensionality of the current operational environment}

Starting from the consideration that in hybrid-type conflicts the conduct of military action involves more than the design of physical combat power and the engagement of conventional forces of the enemy, the operational framework must extend beyond the geographically delimited geographical area in which the battle takes place.

The term "hybrid conflict" / "hybrid war" is used in military literature to define a military strategy in which conventional warfare, unconventional and asymmetric elements are encountered. The multidimensionality of the current operational environment is given by the number and quality of the various actors: state actors, non-state actors, sponsoring states, as well as multiple threats, risks and threats manifested in the physical environment of a conventional nature (conventional military forces in the legitimate service), unconventional (Nuclear Forces, Special Operation ForcesSOF, Chemical, Biological, Radiological and Nuclear Weapons - CBRNW and Industrial Toxic Materials - ITM, Improvised Explosive Devices - IED) and asymmetric (guerrillas, insurgent groups and separatists terrorist and criminal organizations) and virtual cybernetic (informational) environments, all engaged in combat combined and coordinated against a superior enemy in military forces and means.

The geopolitical and geostrategic context, the extent of political, economic, cultural and religious interests, justify us considering that diplomatic, political, informational and economic issues as well as other non-military measures nowadays occupy a special place in shaping the operational environment. Their importance is felt not only during the escalation of the crisis but also in the process of its completion, but even during the military operations, marking the framework of the armed struggle conflict architecture.

The multitude of actors present and involved, along with the diversity and scale of the risks and threats they generate, give rise to a multidimensional operational environment in relation to the five dimensions we were familiar with in the military literature: Earth, Air, Maritime and Cosmic Environment, along with which has been added to the informational environment.

Overlaying these environments and creating an integrated image of the battle space is the conventional or classic operating environment. Is this environment, the multidimensional environment in which the operations are being prepared and executed in a conflict? How has this multidimensionality actually expanded? Yes, the operational environment has expanded beyond classical conceptual boundaries, and we note that the North Atlantic Alliance has included and defined in Article 5 and cyber attack, accepting a new dimension in the operational environment: "We consider cyber defense as part of the task NATO's basic collective defense. A decision to determine the situation where a cyber attack could lead to invoking art. 5 will be taken by the North Atlantic Council on a case-by-case basis" [1].

At the same time, the diplomatic environment, the economic environment and the psychological environment complement the image of the multidimensionality and complexity of the extended framework of operations. The overlapping of these operating environments, like layers, can be described as the hybrid operating environment. Incorporating this situation in an integrated manner that leads to the understanding of 
the effects and to establishing the interdependencies between the existing factors goes beyond the multidimensionality aspects and motivates the complexity attributes.

The assessment of the hybrid operational environment in the area of strategic, operational and tactical interest is achieved by applying scientific instruments and methods to identify its characteristic features.

At the strategic and operative level, the analysis of the conventional operations environment should begin with the analysis of the political environment, interconnections and the diplomatic and economic implications deriving from the modeling or decisive actions. The political environment is often marked by the personality of some leaders and will be decisive in the direction of the evolution of the situation in the operational environment. This requires knowing their personality and estimating the decisions that will be taken to respond to the actions of the potential adversary. The social aspects of modeling actions in the operational environment will be exploited from an informational and media point of view and the effects should serve the conception of their own operations and provide a clear picture and perspective on the predicted effects.

Analysis of the physical environment and the time factor develops at the operative level, but it gains more value at the tactical level. The results of the analysis will be found in the estimates of the battlefield military factors identifying the importance of the key points in the field, the access routes, the existence of obstacles and possibilities of masking / hiding, the situation and the possibilities of the enemy's own and the forces, the situation of the civilians. All these aspects will be correlated with the time limit available to the mission, resulting in a comprehensive approach to the operational environment.
Understanding the Hybrid Operating Environment is a requirement of success for military operations. Methods and tools used to understand the hybrid operational environment: Operational level, analysis of operational variables - PMESII-PT (political, military, economic, social, information infrastructure, physical environment, time), ASCOPE, organizations, people and events); at the tactical level to analyze the OAKOC geographic environment (obstacles, avenues of approach, key terrain, observation and fields of fire and cover and concealment), along with mission variables, METT-TC (mission, enemy, terrain and weather, support available, time and civil considerations, and SWEAT-MSO (sewer, water, electricity, academic, trash, medical, security and other) for basic needs analysis. Operational level analysis tools can also be applied at the tactical level.

The operational environment becomes a hybrid environment by exploiting cultural, traditions and history. Informational and psychological operations aimed at forming a dedicated opinion or informally supporting a hybrid adversary will use these media to disseminate specific messages.

\section{The role of military scenarios in the design of military actions}

Hybrid threats from the operating environment are particularly complex, always different, tailored to the vulnerabilities of the target actor, producing imbalance effects that weaken its capabilities, diminishing its power to respond. Combating the hybrid threat thus becomes one of the most complex issues for which it is necessary to find the right answers.

Given the complexity of the operational environment and its high level of uncertainty, identifying and counteracting the hybrid threat and addressing the effects it generates can be achieved through a proactive approach, grounded in a 
prospective way of thinking anticipating the likely actions of the opponent.

The analysis of the operational environment, the actors involved and the relationships between them, as well as the strategies they aim at achieving the goals pursued, is a mandatory condition for the preparation of force and the planning of actions. The most effective way to achieve this analytical process and to use the results obtained is to include it in the scenarios as likely or desirable dynamics of the situation in which it operates.

The scenarios describe a number of future conditions, opportunities, threats and obstacles that influence the possibility of achieving the proposed objectives. They direct how to approach future situations and implicitly how to act. The commander must anticipate the evolution of the current situation or the configuration of possible situations and plan the actions necessary to counteract the hypothetical threats.

The script is also an excellent tool for conducting training activities. Exercises at any level of military art for the purposes of learning, training, evaluation or rehearsal prior to the execution of a mission imply the existence of a basic operational framework, described by the scenario. It fits and directs the training process to the smallest detail, thus making a substantial contribution to achieving the goals.

The scenario is characterized by the fact that it has a descriptive approach to a situation of crisis or conflict, with details corresponding to the level at which the scenario (strategic, operative or tactical) is designed. The purpose of these scenarios is to train / verify the capabilities of the participating structures in the exercise of the dynamic planning process during the fight. In this situation, the planner of the exercise must draw up a conception of the operation.

This conception is the basis for drawing up the management and information documents issued for the trained / evaluated structure. The trained / evaluated structure needs to adapt its own design to follow the design of the required operation. Thus, the structure being evaluated is channeled to go through the situations and moments set by the planners of the exercise, in order to solve them and achieve the objectives. From this point of view, the use of constructive simulation is very effective. The constructive simulation can generate concrete situations, close to the real operational environment, which the exercise planner omits or erroneously developed.

The military scenario must have a configuration setting out key elements that define the operational environment and its evolution over time. He also has to provide all the information necessary for carrying out the planning of operations.

The architecture of NATO-based scenarios in modules is presented in the specific collective training documents and in the Driving Training / Mission Training [3] guidelines as follows:

Module 1. Geostrategic Situation - it includes a general description of the crisis area, including the main regional actors, the presentation of the crisis, the historical aspects, the main political, economic, cultural, humanitarian and legal conditions, as well as the membership in different treaties and alliances which regulates the relationships between actors.

Module 2. Operations Theater - provides operational theater data and information to support situation assessment and operation planning, operational theater data, information or country studies, and campaign plan for the opponent.

Module 3. Strategic Initiation - includes the ultimate internationally desirable political status, objectives, limitations and directions as well as strategic assessments and planning directions specific to NATO's crisis response system.

Module 4. Crisis Response Planning Information - includes updated data and information on the regional and international situation, the situation and 
layout of its own forces, data and information on civilian-military relations, data on civil-military cooperation, environmental assessment, etc.

Module 5. Information on force deployment and deployment - includes data and information necessary to achieve operational and force deployment planning, allied forces list, results of force balance analysis, specific information on treaties and armaments control agreements that may impact on the exercise, the list of targets at the common level, the authorization regarding the rules of engagement/ implementation of the engagement rules.

Module 6. Implementation information includes the following: the road to crisis (brief outline of the main events that led to the configuration of the current situation); synthesis and report of information; estimates and operational reports - shall be transmitted before and after the start of the exercise; Battle order / Transfer of authority / Force status; data and information on the common operational image for the area of interest.

The scenario is not a prediction of the future, most of the time the product resulting from the scenario is to be seen as a sum of solutions and not as a summed solution. The scenario creates the operational framework and the opportunity that the objectives generated by the need to manage the risks that may arise during missions meet the competence, professional experience and imagination of the screenwriters, the lessons learned from the previous operations and the precepts that guide the implementation of all branches of military science.

The scenario method can also be used to assess the ability to apply the fighting power of its own forces, to determine the efficiency of designing forces structures to carry out operations, especially in the hybrid operating environment. In this sense, scriptwriters can alter a wide range of features of artificially created environment for action, so as to cover as many forms and intensities as possible for the hybrid threat.

Another purpose of using the scenario method is to test some tactics, techniques and procedures (TTP) for action or weapon systems (or procedures for using them) that are or are to be put into use. In this regard, the most effective ways of adjusting possible malfunctions are identified.

Another way of using the scenario method is to involve some structures in the planning process. The goal is to create models to solve the various surprising situations where proactive thinking of planners can use to develop and apply the solution. These scenarios help to increase the speed of response in critical situations and increase efficiency in decision-making in unpredictable situations.

The process of designing and developing scenarios is extremely complex and requires considerable effort on the part of all involved. The role of military scenarios is to create a sequential, dynamic image of events from a hypothetical situation in order to determine the recipient to formulate responses.

The multidimensionality feature of the crisis situation in the hybrid operating environment broadens the spectrum of threats that can be included in the scenario. The situation calls for the most appropriate response to be identified by developing existing capabilities, creating new ones, and finding ways to link each other to address the specific threats of this type of environment.

The scenario method aims at eliminating uncertainty or at least setting controllable limits around the uncertainties generated by the hybrid operating environment and concentrating planners' efforts on addressing urgent planning requirements. Scenarios are thus extremely useful tools for increasing the efficiency of the decisionmaking process according to the evolution of the operational environment. 


\section{Conclusions}

The ambiguity and uncertainty of the current operational environment creates the need for an extremely complex analysis to prepare a military force capable of effectively achieving success in the operation. Following the assessment of the current operational environment we have found that the influence of economic, political, diplomatic, informational and psychological aspects now occupies, more than ever, a very important place in the military operations architecture, but also that the evolution of the technology in the field of communications, and cyber defense has led to an exponential increase in the power of information.

The comprehensive approach to the current, complex and dynamic operational environment, marked by instability and insecurity, leads to the conclusion that it meets the characteristics of a hybrid knowledge based environment that integrates and interconnects physical, informational, cybernetics, psychological, media, politics, economics and infrastructure, in which a multitude of actors act and a wide range of risks and threats are manifested.

I felt the need to address the multidimensionality of the modern operational environment by highlighting that, from a doctrinal and theoretical point of view, Land Forces - are planning and carrying out actions in view of the multitude of threats present in the independent, integrated, integrated and multinational operational environment.

A very important aspect that differentiates the hybrid threat from the sum of the threats present in the military conflicts of the past is that in the first case, the classical, conventional, conventional armed struggle between two armed forces passes secondarily or may be absent perfection. In other words, in the hybrid operating environment, the share of actions, from a typology perspective, shows a migration from regular to unconventional, especially to asymmetric ones.

Chances of success in confronting a hybrid enemy are significantly augmented by the use of all power, political, military, informational, economic and legislative instruments and by identifying and exploiting its vulnerabilities. Other important ways to augment the capabilities required to combat hybrid threats are to predict the crisis situations that may occur and to properly train forces with such a destination. These two aspects are linked to each other through an effective planning process that must be carried out at all levels of the structures involved in the conflict.

\section{References}

[1] Statement of the NATO Summit in Wales, 4-5 september 2014, Art 72, consultedon https://fcnap.ro/declaratia-summit-ului-nato-din-tara-galilor/, accessed on 28.02.2017.

[2] Dan Lucian PETRESCU, The military scenario, the fundamental conceptual framework for the exercises at the tactical-operative level,in the National Defense University Bulletin,,Carol I" March, 2015, p. 218.

[3] ***BiSC 075-003, Collective training and exercise directive (CT\&ED), 2013, appendix 1 to Annex $\mathrm{M}$ available onwww.act.nato.int/images/stories/structure/jft/bi-sc-753 final.pdf, accessed at26.01.2018. 